\title{
Resources Scheduling Algorithm in Power Wireless Private Network Based on SDON
}

\author{
Ningzhe Xing \\ State Network Hebei Electric Power Co., Ltd., Information and Communication Branch, Beijing, China \\ Correspondence should be addressed to Ningzhe Xing; xingningzhe@163.com
}

Received 24 March 2017; Accepted 30 July 2017; Published 12 September 2017

Academic Editor: Hui Yang

Copyright (c) 2017 Ningzhe Xing. This is an open access article distributed under the Creative Commons Attribution License, which permits unrestricted use, distribution, and reproduction in any medium, provided the original work is properly cited.

With the increasing of the business carried by the power wireless private network and the continuous development of the new network technology, the problems in the traditional mode of power wireless private network are becoming more and more obvious. In order to solve the problems such as poor business service quality, low system throughput, and fairness problems in the uplink resource scheduling process of power wireless private network, a dynamic uplink resource scheduling algorithm is proposed on the basis of Software Defined Optical Network (SDON). By studying the business characteristics of the uplink transmission of power wireless network, the priority of the service is evaluated before the resource scheduling is carried out. According to the characteristics of OFDM resource allocation and the numerical control separation and programmable feature of SDON, different scheduling methods are designed for different services. Simulation analysis shows that this algorithm can effectively improve the system throughput, guarantee QoS, and improve the transmission performance of different services.

\section{Introduction}

With the development of smart distribution grid, the application of new generation wireless broadband technology in distribution network has become the research hotspot in current power communication system $[1,2]$. At present, the power distribution and utilization network has characteristics of complex structure, excessive devices, wide distribution of business nodes mostly in dense urban areas, poor operating environment, long communication distance, high cost of laying optical cable, and difficulty of construction, resulting in the difficult selection of power network communication technology.

Time Division Long Term Evolution (TD-LTE) is the mainstream of wireless broadband access technology. In smart grid, TD-LTE is used to build power wireless broadband network, which will be an important way of distribution network communications [3, 4]. TD-LTE for the construction of power wireless private network can provide with large capacity, high bandwidth, low latency, multilevel QoS protection, and other significant features of the communication network. Therefore, the study of TD-LTE technology applying in power wireless private network under SDON architecture is of great significance. With the large-scale commercial deployment of LTE networks, the new architecture of BBU centralized deployment model came into being. In this architecture, the construction of the fronthaul network has put forward the three requirements of high bandwidth, low delay, and high precision [5]. For this reason, OFDM-PON is utilized as a fronthaul network carrying LTE. OFDM-PON connects an LTE base station and a BBU by optical fibers, bearing the radio access in the power distribution network. As one of the next-generation fiber access networks, the OFDM-PON system has the following advantages comparing with other PON systems: high spectrum utilization, strong antidispersion capability, low cost, and flexible resource modulation and distribution mechanism [6]. In addition, with Radio over Fiber (RoF) technology applied in this fronthaul network, radio signal can be transmitted directly through OFDM-PON [7]. This combination of wired and wireless technology complementing each other has the advantages of low loss, long transmission distance, high bandwidth, strong anti-interference ability, flexible operation, and dynamic configuration of resources.

However, as an optical fiber communication, OFDMPON is difficult to break the Shannon limit by enhancing 
TABLE 1: The QoS requirement of business.

\begin{tabular}{|c|c|c|c|}
\hline Business class & Typical business & Delay requirement & $\begin{array}{l}\text { Bandwidth requirement of an } \\
\text { access node }\end{array}$ \\
\hline \multirow{2}{*}{ Power distribution } & Distribution automation & $\leq 1000 \mathrm{~ms}$ & $20 \mathrm{kbps}$ \\
\hline & Distributed power $(10 \mathrm{kV})$ & $\leq 1000 \mathrm{~ms}$ & $2 \mathrm{kbps}$ \\
\hline \multirow{3}{*}{$\begin{array}{l}\text { Distribution network } \\
\text { operation monitoring }\end{array}$} & Transmission line monitoring (video) & $\leq 1 \mathrm{~s}$ & $2 \mathrm{Mbps}$ \\
\hline & Mobile inspection (video) & $\leq 10 \mathrm{~s}$ & $384 \mathrm{kbps}$ \\
\hline & Infrastructure video monitoring & $\leq 1 \mathrm{~s}$ & $2 \mathrm{Mbps}$ \\
\hline \multirow{2}{*}{ Power utilization } & Electricity information collection & $\leq 10 \mathrm{~s}$ & $10 \mathrm{kbps}$ \\
\hline & Electric vehicle charging pile & $\leq 1 \mathrm{~s}$ & $0.3 \mathrm{kbps}$ \\
\hline
\end{tabular}

fiber capacity. And expanding the scale will bring the cost and energy consumption of the sharp rise, so its development needs to find a new breakthrough point. SDON networking can solve the above problems fundamentally. By introducing a software defined intelligent network in the optical layer, the PON network can be made more active, thus further releasing its bandwidth potential and promoting the deep integration of network and business [8]. SDON network architecture and SDN are basically the same. Only the infrastructure layer of the network equipment correspondingly by the IP network switches into OTN and other types of equipment. In order to fit the underlying optical network, the interface protocol also needs to be extended on the basis of the OpenFlow protocol.

In both OFDM-PON and the LTE network carried by it, multicarrier technology is utilized in the uplink and downlink transmission schemes and the uplink is SC-FDMA, while the downlink is OFDMA. Multicarrier means that the channel is divided into a number of orthogonal subcarriers, and different carrier frequency modulation methods are used in each subcarrier; thus the signal with high bit rate is able to be converted to several low bit rate signals for transmission. High frequency is used in power broadband wireless private network; specifically the same frequency networking technology is used in the $1.8 \mathrm{GHz}$ band. However, only $5 \mathrm{M}$ bandwidth can be applied in the construction of power wireless private network. So it is more necessary to focus on the improvement of spectrum utilization and optimization of uplink and downlink resource reuse [9]. Considering the characteristics of the uplink business data in the power grid, this paper designs a traffic priority based uplink resource scheduling scheme based on the SDON architecture in the power wireless private network [10].

There are many traditional resource scheduling schemes. At present, the uplink scheduling algorithm is based on the Proportional Fair (PF) algorithm. The main problem of this algorithm applied for smart gird network is that they do not consider the actual power grid business situation and cannot guarantee business quality of service (QoS) [11]. In this paper, based on the traditional PF, combined with the priority of the power communication network service, we introduce the SDON technology and design a dynamic uplink scheduling algorithm to ensure the optimal allocation of resources and improve the uplink throughput of the system [12].

\section{Power Wireless Private Network Uplink Communication Requirements}

Playing a supporting role, the power communication private network is required to be able to cover all aspects of the power system, including power generation, transmission, distribution, utilization, and scheduling process. For the guarantee of the power communication, businesses such as metering, online monitoring, video management, and distribution automation need to be better carried out. The coverage and communication requirements of various types of services in power distribution communication network are different. It is necessary to focus on the analysis of these requirements, so as to provide the basis for technology and solution selection in the power distribution communication network construction.

2.1. Demand Analysis of Power Distribution and Utilization Business Uplink Transmission. The typical business in power distribution network includes traditional business such as distribution automation (remote measure, remote control, and remote communication), electricity information collection, transmission line monitoring, and infrastructure video monitoring and new business like distributed power supply $(10 \mathrm{kV})$ and electric vehicle charging pile [13, 14]. As the downlink business volume is smaller than the uplink in the power distribution communication network, this paper focuses on the carry of uplink business in power wireless private network. According to the function, uplink business can be classed into power distribution, power utilization, distribution network operation monitoring, and other types. The QoS requirements of some typical services in the power distribution network are listed in Table 1.

2.2. Priority of the Business in Power Distribution Network. According to the QoS requirements and the importance of the business, the priority of key business transmitting in the power wireless private network is divided into three categories:

(1) The first class is the power distribution business: the bandwidth requirements of such services are usually low, but the delay requirements are relatively high. The typical business is distribution automation. With distribution automation system as the core, it is 
a comprehensive usage of various communication methods to achieve the monitoring and controlling in distribution network (including distributed power and micronetwork). Through the information integration with relevant application systems, distribution automation can achieve the scheduling command and scientific management of distribution network. Power distribution business is closely related to the electricity production. It requires high reliability and real-time of communication and has strict requirements on transmission delay and loss. However, its traffic is generally small.

(2) The second class is distribution network operation monitoring business: it includes video monitoring, transmission line monitoring (video), mobile office and inspection (video), and other services, for instance, unattended substation monitoring, accident repair site analysis, and important switching equipment monitoring. Power company monitoring center needs to manage all the video information of the substation. Although the data amount of such business is large, it can tolerate a larger transmission delay.

(3) The third is power utilization business: this class of business is the real-time monitoring, collecting, and processing of electricity information of users. Its functions are automatic collection of electricity information, abnormal measurement detection, power quality test, and analysis and management of power consumption. The business data is mainly collected from the electricity collection terminals. And the main collection methods are random call and active reporting, from which the regularly automatic collection is commonly used. And round robin method is used for the scheduling of the concentrator. So both the delay and bandwidth requirements for this class of business are not high.

Based on the QoS requirements of uplink business in the power distribution network, this paper designs a dynamic resource scheduling algorithm based on SDON for the purpose of optimal transmission and resource allocation.

\section{Network Architecture Based on SDON}

3.1. Advantages of SDON Applied in Power Grid. SDON technology arranging and abstracting the network resources through the controllers breaks the way of traditional networking and makes the realization of customer-centric application possible $[12,15]$. The grid can rely on the programmable features of SDON technology and flexible resource scheduling and abstraction ability to realize the rapid distribution of power grid service, flexible adjustment of bandwidth, and intelligent perception of service quality, so as to provide users with faster, more flexible and smarter green business. SDON technology has three main advantages:

(1) SDON is able to virtualize the management of optical network resources. Through a unified and open resource management platform into the network infrastructure management, make full use of the advantages of various types of resources to achieve optimal resource utilization.

(2) Optical network resources can be programmed on demand. Optical network resources can be programmed on demand control and offer the fastest and most flexible way to provide users with customized service functions and enhance customer satisfaction.

(3) Multilevel multivendor network can be achieved between the more convenient interoperability. Through the expansion of OpenFlow and other related protocols and the development of the objectoriented interactive control interface, it can achieve heterogeneous network cross-level interconnection. Thus in the data network and optical network, core network and access network, wireless network, and cable network establish a unified control of the cross-domain interoperability network architecture.

3.2. Network Architecture. In the architecture shown in Figure 1, SDON is introduced into the power wireless private network to control the resource scheduling process on the link between BBU and RF antennas. And we use OFDMPON to carry this data transmission. That means OFDMPON works as the fronthaul network of the wireless network in power grid. Furthermore, the signal transmitting in the PON is processed using RoF technology. Thus even though the transmission medium is fiber, the transmitting signal in OFDM-PON is still radio frequency. It is obvious that this architecture is a comprehensive use case of three technologies, combining their advantages in capacity, flexibility, and antijamming ability.

\section{Resource Scheduling Model}

4.1. Advantages of LTE in Power Grid. LTE technology has advantages of high bandwidth and low latency. Business data transmission system based on TD-LTE technology can adapt to the complex structure and wide demand characteristics of power grid:

(i) Due to its high data throughput and high spectrum utilization, TD-LTE can provide a higher transmission rate for power grid data transmission in the case of limited bandwidth resources.

(ii) TDD duplex mode is used in TD-LTE technology. This makes it flexible to change the ratio of uplink and downlink resources. So TD-LTE can meet the special requirement in power system that the uplink demand rate is higher than the downlink. Thus it can be better applied to the power communication network construction [16].

As shown in Table 2, the frame structure with uplink slots occupying up to 75 percent is preferentially selected by this method.

4.2. OFDM Resource Allocation Model. The basic idea of OFDM divides a high-speed serial data stream into a plurality 


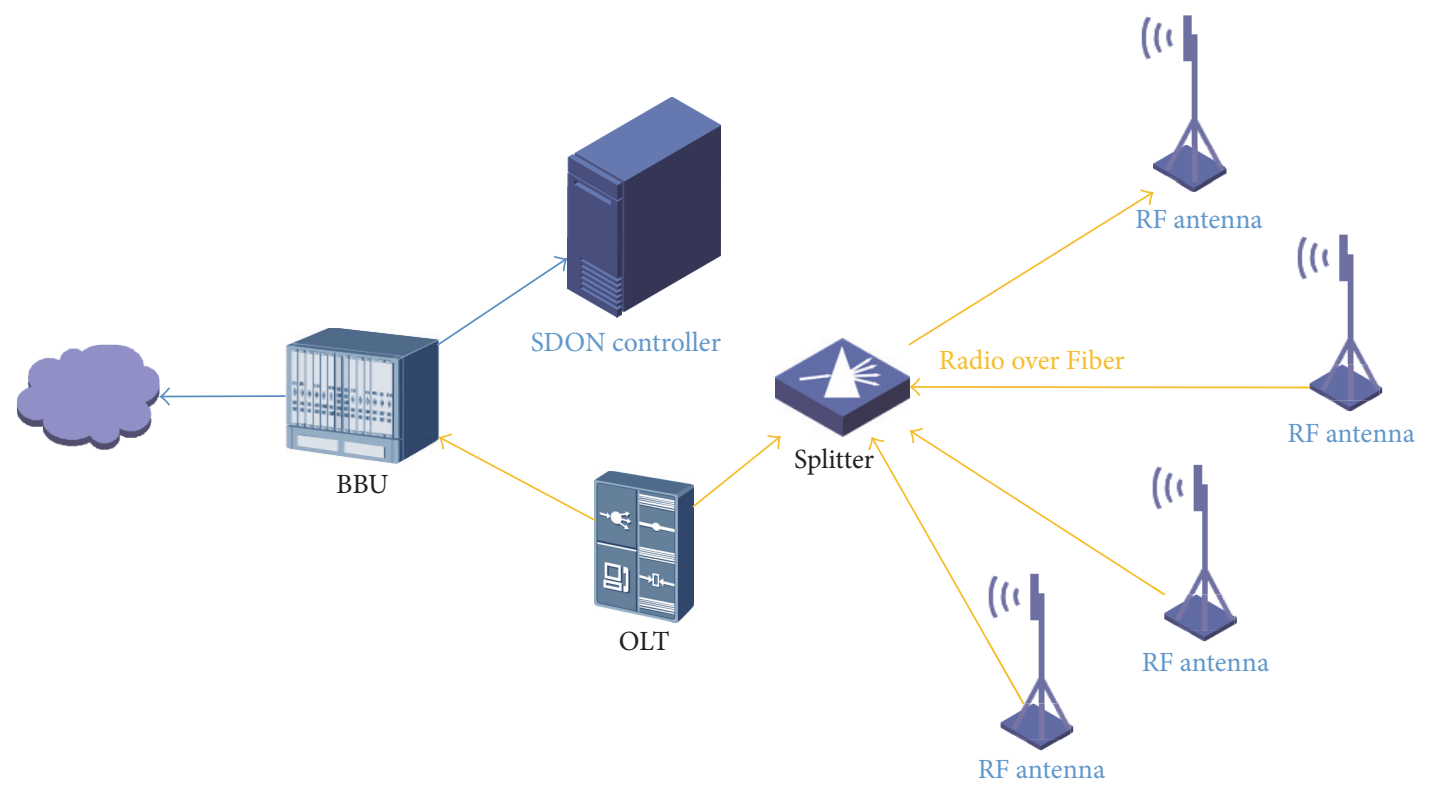

FIgURE 1: SDON based network architecture.

TABLE 2: Uplink and downlink ratio.

\begin{tabular}{llllllllll}
\hline \multirow{2}{*}{ Frame } & & \multicolumn{9}{c}{ Subframe number } & & & \\
& 0 & 1 & 2 & 3 & 4 & 5 & 6 & 7 & 8 \\
\hline $5 \mathrm{~ms}$ & $\mathrm{D}$ & $\mathrm{S}$ & $\mathrm{U}$ & $\mathrm{U}$ & $\mathrm{U}$ & $\mathrm{D}$ & $\mathrm{S}$ & $\mathrm{U}$ & $\mathrm{U}$ \\
\hline
\end{tabular}

of low-speed data streams and transmits the plurality of lowspeed data streams through subcarriers orthogonal to each other in several frequencies, thereby effectively improving the bandwidth utilization efficiency of the system.

Since RoF is used in OFDM-PON to transmit radio frequency signal according to the proposed architecture shown in Figure 1, we mainly focus on the resource allocation in LTE network, which is also an OFDM process. The physical layer frame structure of TD-LTE system is as follows: the $10 \mathrm{~ms}$ TD-LTE radio frame contains two equal fields, the length of which is $153600 \cdot T_{s}=5 \mathrm{~ms}$. And each field contains five subframes; the length is $T_{t}=30720 \cdot T_{s}=1 \mathrm{~ms}$. And each subframe contains two slots; the length of each slot is $0.5 \mathrm{~ms}$.

For TDD, the uplink and downlink are separated in time, while the carrier frequency is the same. That is, in every $10 \mathrm{~ms}$ cycle, there are totally 10 subframes using for uplink and downlink, and each subframe is either uplink or downlink.

In TD-LTE communication system, the minimum resource allocation unit is defined as 12 consecutive subcarriers in the frequency domain and 7 OFDM symbols in the time domain (in the conventional CP case). That is the resource block (RB). For each user, one or more RBs can be used for business bearing. Figure 2 shows an OFDM downlink RB schematic.

\section{Uplink Scheduling Algorithm Design}

5.1. Traditional Packet Scheduling Algorithm. The traditional packet scheduling algorithms include Maximum Carrier to Interference (MAX C/I) algorithm, Round Robin (RR) algorithm, and Proportional Fair (PF) algorithm. The goal of the MAX C/I algorithm is to obtain the maximum throughput of the system, so it does not take into account the fairness of the user transmission. The Round Robin algorithm takes into account the fairness of the users and transmits the user data in turn but sacrifices the throughput of the system. The Proportional Fair algorithm is between those two algorithms, taking into account both fairness and system throughput. So many resource scheduling systems choose the $\mathrm{PF}$ algorithm $[17,18]$.

However, due to the lack of business QoS requirements and the need for uplink resource allocation fairness considerations, the above three algorithms are not applicable to the power wireless network resource scheduling system. Based on the business characteristics of power grid, this paper designs an algorithm of uplink dynamic scheduling based on PF algorithm, which taking into account the power grid business QoS requirements, the terminal channel quality, and a series of key factors. At the same time, SDN technology is introduced to manage OFDM-PON resources. The tasks are managed according to the priority of the software, and the resources are allocated based on the different priorities of the tasks, which makes the uplink resource scheduling more fast, flexible, and intelligent.

5.2. Uplink Dynamic Scheduling Algorithm Based on Service Priority. As show in Figure 3, the whole algorithm is divided into two parts: first of all, for all users to apply for data, SDON management platform according to different types of business using different scheduling algorithms to determine 


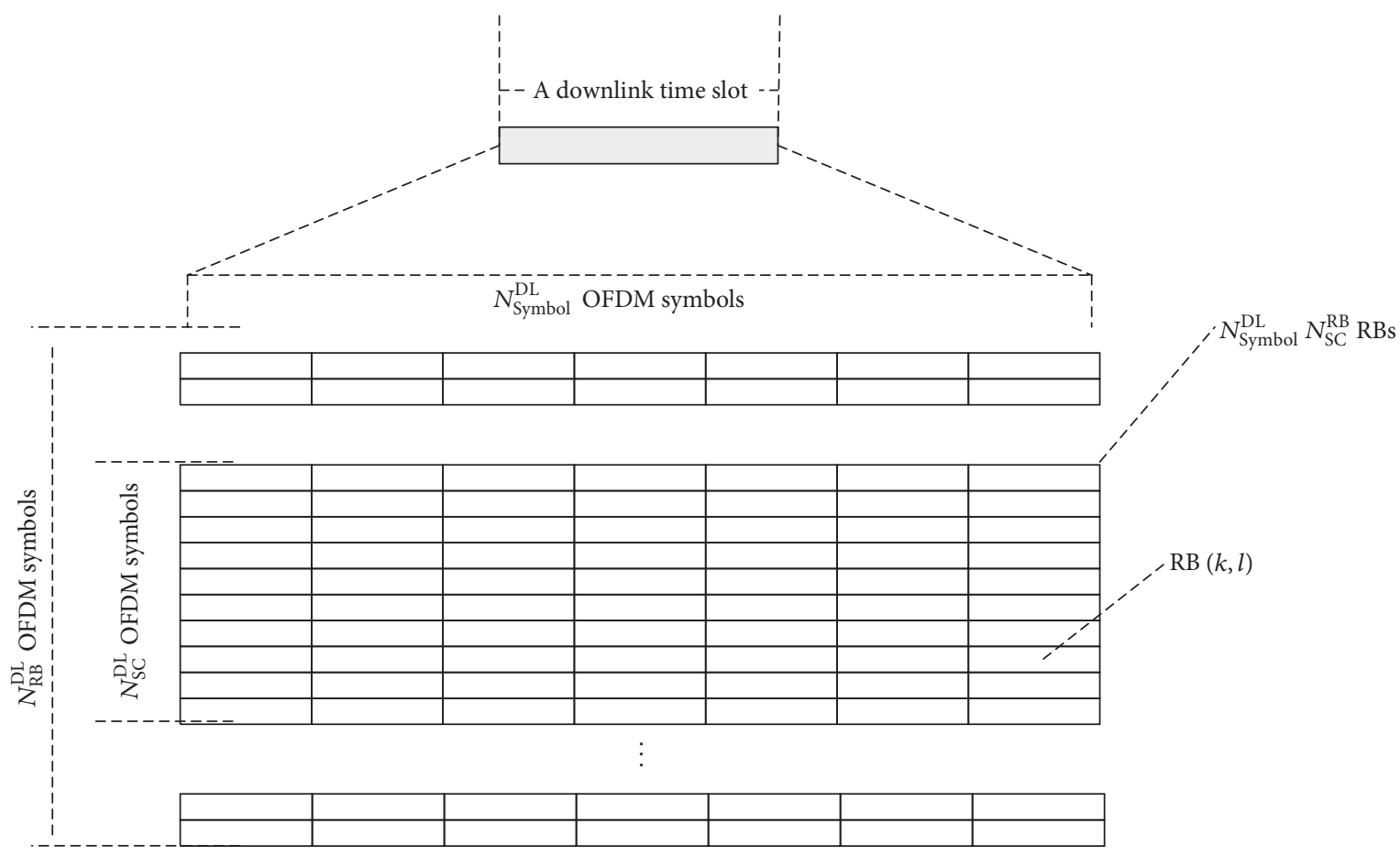

FIgURE 2: Schematic of RB in OFDM.

the task priority, and then through the SDON resource management platform for the priority of the task of the business resource allocation.

Different scheduling methods for each type of business are as follows.

(1) For the first class of distribution business $\phi_{1}$, due to the strong real-time requirements and high security requirements, we need to ensure that the packet can not be lost and can reach the destination on time. Obviously, it can be found from (1) that the packet will get the higher priority if life cycle is shorter and the data of the packet is bigger.

$$
k_{1}=\arg \max _{k \in \phi_{1}} \frac{T_{k}}{S_{k}} .
$$

Select the business packet $k_{1}$ with highest priority, where $k \in \phi_{1} . T_{k}$ indicates the life cycle of the packet. $S_{k}$ indicates the size of the packet. When selecting a channel, use the following formula:

$$
n_{1}=\min _{n \in \Omega}\left\{T_{k 1}-\frac{S_{k 1}}{C_{k 1, n}}\right\},
$$

which requests $T_{k 1}-S_{k 1} / C_{k 1, n}>0$, where $C_{k 1, n}$ indicates the rate that $k_{1}$ packet is allocated on the $n$th packet, and then update $\phi_{1}=\phi_{1} / k_{1}, \rho_{k 1, n 1}=1 . \rho_{k, n}=1$ indicates that the $k \mathrm{th}$ packet is allocated on the $n$th resource block.

(2) For the second type of distribution network operation monitoring business $\phi_{2}$, real-time requirements are not as high as the first class of business. As the current network uses video surveillance, bandwidth demand is greater and requires as much as possible to reduce the data packet loss rate (PLR); we can select the highest priority business packet through the formula:

$$
k_{1}=\arg \max _{k \in \phi 2} \frac{T_{k}}{T_{n}^{\mathrm{req}}}
$$

where $T_{n}^{\text {req }}$ represents the waiting time threshold on the $n$th resource block; the formula which is used to find the optimal packet allocation method is as follows:

$$
n_{1}=\max _{n \in \Omega} \gamma_{k 1, n}
$$

Then update $\phi_{2}=\phi_{2} / k_{1}, \rho_{k 1, n 1}=1$.

(3) For the third type of the electricity business $\phi_{3}$, such business does not have strict real-time requirements, only need to do their best to complete, and send as much data as possible to the destination. The formula below is used to find the highest priority:

$$
k_{1}=\arg \max _{k \in \phi 3} \frac{r_{k}(t)}{\overline{r_{k}}(t)},
$$

where

$$
\overline{r_{k}}(t)=\left(1-\frac{1}{T}\right) \overline{r_{k}}(t-1)+\frac{1}{T} \log _{2}\left(1+\gamma_{k, n}\right)
$$

indicates the transmission rate of the $k$ th packet at time $t, \bar{r}_{k}(t)$ is updated once in each time slot, $\gamma_{k, n}$ represents the signal-to-noise ratio of the $k$ th packet transmitted at the $n$th resource block, and the parameter $T$ is the sliding window size. The setting of the value $T$ should balance both throughput and fairness. Find the optimal packet through (4) still; then update $\phi_{3}=\phi_{3} / k_{1}, \rho_{k 1, n 1}=1$. 


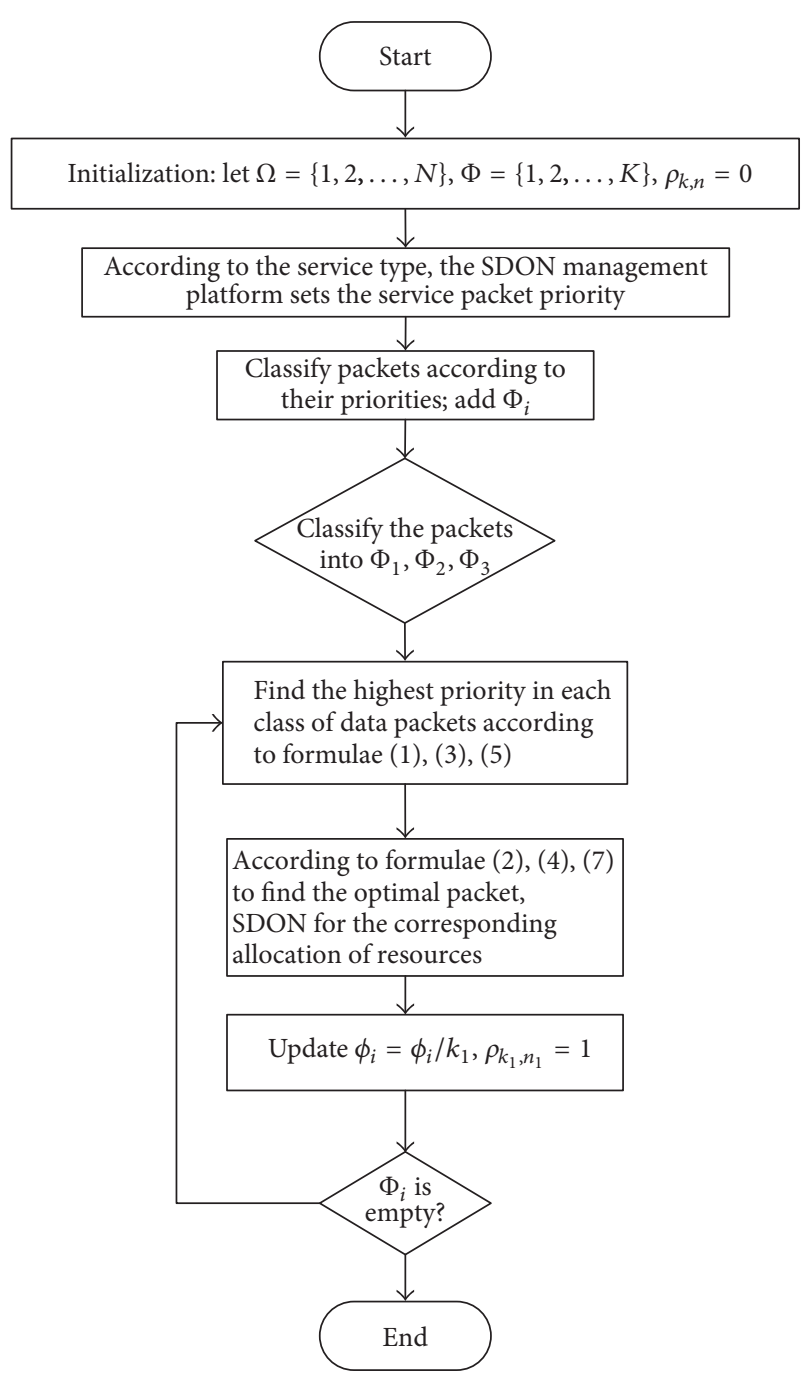

FIGURE 3: Structure of uplink dynamic scheduling algorithm.

\section{Simulation and Result Analysis}

In order to simulate the business data in the power grid, the simulation will randomly generate different kinds of business data packets in each TTI and record the simulation data in the process of execution. As the algorithm is based on the business priority to dynamically schedule the packet, in order to evaluate the advantages and disadvantages of the proposed algorithm, we compare the overall throughput and the throughput of different service packets with the traditional PF algorithm and the RR algorithm.

6.1. System Throughput. The simulation compared the throughput of the system under the new algorithm proposed by us and the traditional algorithm. The data include the throughput of the three different algorithms when dealing with different data requests. By setting the number of data requests initiated by the system unit time, record the amount of business data successfully transmitted under different algorithms per unit time. The minimum allocation unit of

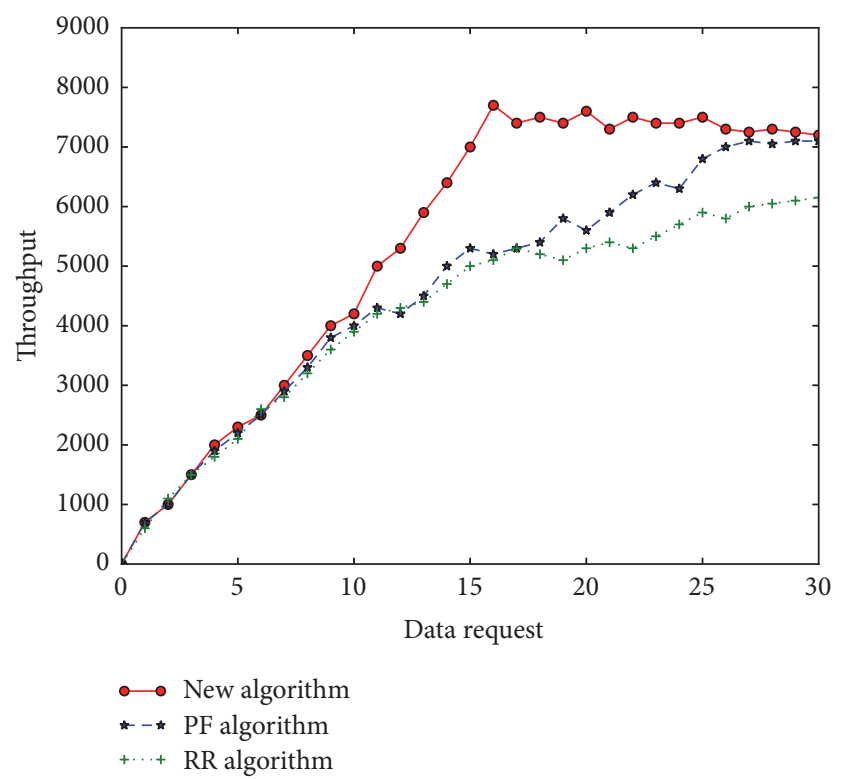

FIGURE 4: System throughput under different algorithm.

the data in the simulation is the resource block $\mathrm{RB}$ defined in TD-LTE. RB is composed of 12 consecutive subcarriers and 7 OFDM symbols in the time domain. The results are shown in Figure 4.

Compared with the traditional PF and RR algorithms, the throughput of the new algorithm will be higher. Particularly when the number of packets is close to the maximum load of the LTE unit frame, the gap between the proposed algorithm and the traditional algorithms is the largest. As the frame transmission capacity tends to saturate, the gap between them eventually decreases. It can be seen from the figure that the new algorithm proposed by us has better performance in data transmission capability.

6.2. System Fairness. We use a measure of scheduling fairness of the indicators Jain Fairness to a variety of algorithms which were compared; the Jain Fairness calculation method is as follows:

$$
I_{\text {Jain }}=\frac{\left|\sum_{i=1}^{n} x_{i}\right|^{2}}{n \sum_{i=1}^{n} x_{i}^{2}},
$$

where $n$ represents the number of users, $x_{i}$ represents the total number of resources allocated by user $i$, and the larger the value of Jain FI, the more equitable the allocation and the more average the user gets. The fairness index is shown in Figure 5.

As can be seen from the figure, when the simulation has just begun, because the system is in the initialization state, send buffer and receive buffer data are not saturated, so the data has not yet arrived at the receiver. At this time, the PF algorithm and the RR algorithm increase the total data of the denominator when measuring the fairness, but the user's acceptance of the data has not been increased, so the value is reduced, but as the simulation progresses, the experiment 


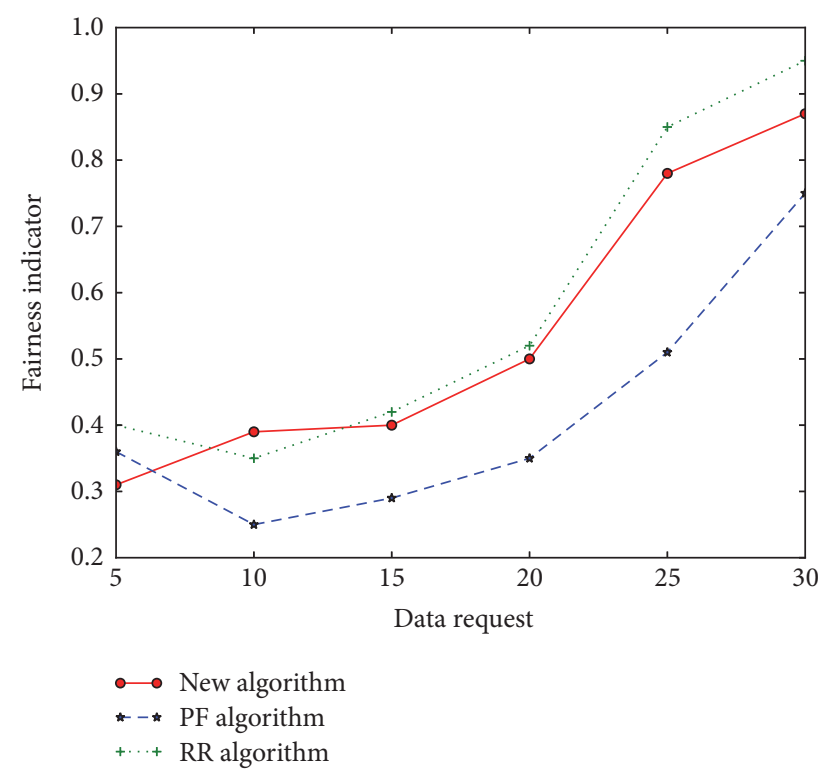

FIGURE 5: System fairness under different algorithm.

tends to be stable and the result shows that the RR algorithm has the highest fairness because it is round robin scheduling. It is shown that the fairness coefficient of the new algorithm is between the traditional PF and RR algorithms. This is because the new algorithm proposed in this paper takes the priority factors of various services such as RLT into account, while the traditional PF and RR algorithms are based on the overall system fairness and do not take the priority of the business on a particular packet into account, so the fairness coefficient is lower.

6.3. The Throughput of Different Business. As the algorithm focuses on the transmission of different priorities of the business, we compare the business data throughput of it with the traditional scheduling algorithm with different priorities. This algorithm divides the service type into three priorities. By counting the amount of different business data for 40 TTIs and comparing them to traditional algorithms. Due to the different bandwidth requirements of each service, in order to visualize the transmission capability of the algorithm for different priority services, we limit the number of packets sent by various services when performing traffic simulation. As shown in Figure 6. As the RR algorithm is round robin scheduling, so the three types of business throughput are basically the same. Because PF algorithm takes the priority and maximum throughput into account, compared with the $\mathrm{RR}$, the throughput of the first and second class business, which owns the higher priority than the third class business, has been improved. In this paper, the proposed algorithm is designed to meet the QoS requirements of different types of services. From the comparison with the results of traditional $\mathrm{PF}$ algorithm, the algorithm can ensure that both of them are transmitted at a higher rate for 1 and 2 categories of business which are with higher priority, at the expense of category 3 business with low-priority. This is expected to meet the priority of high priority traffic transmission, so the algorithm

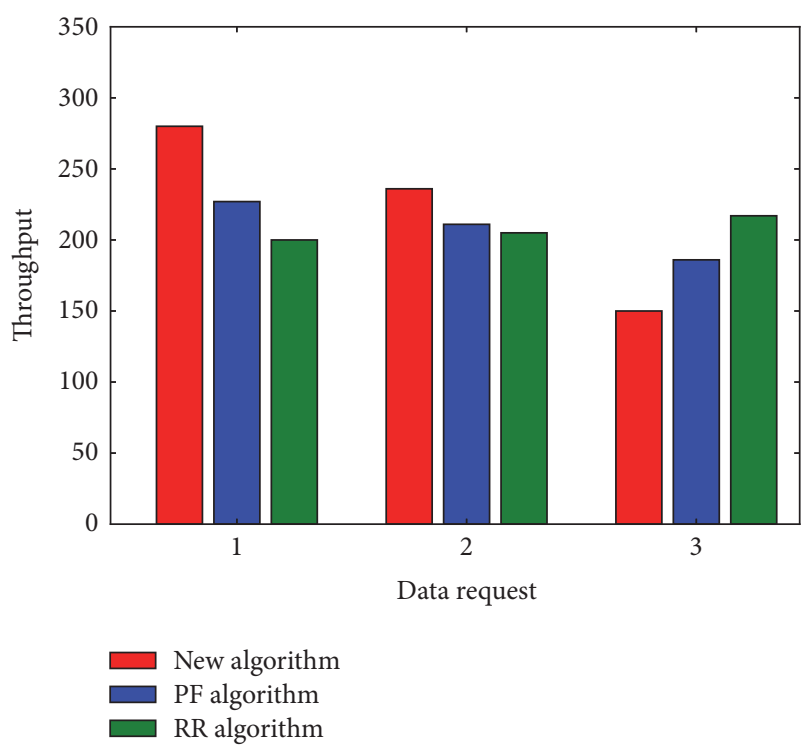

Figure 6: The throughput of different business under different algorithm.

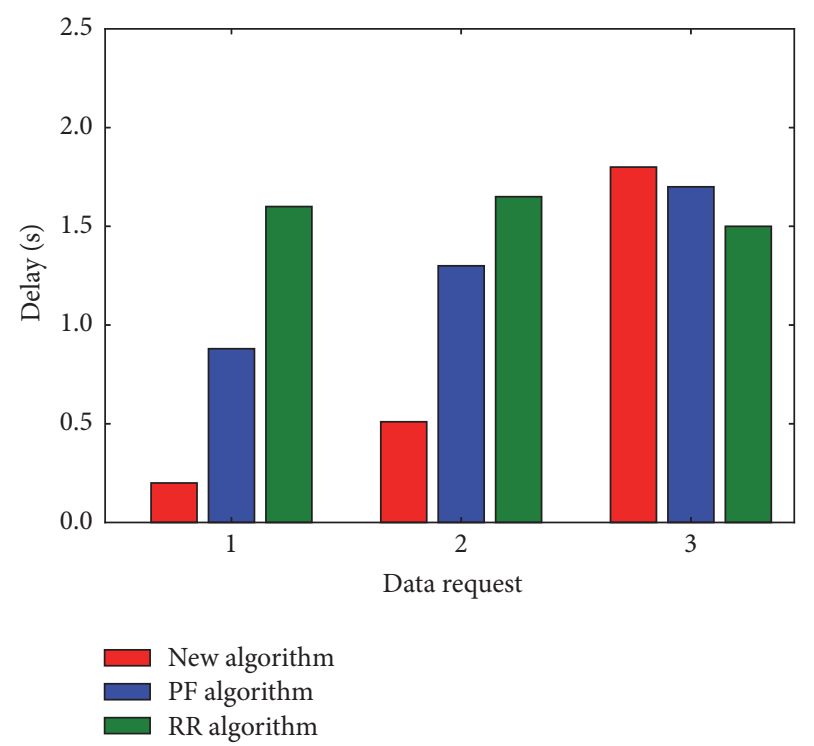

FIGURE 7: The delay of different business under different algorithm.

not only considers the increase in system throughput but also takes into account the priority transmission of high priority business.

6.4. System Delay. In order to verify the guarantee of realtime requirement of the first and second class services, the system simulates the time of various types of packets from generation to the scheduled time under different algorithm as shown in Figure 7. RR algorithm is the round robin scheduling, so all kinds of business scheduling time are basically the same, which led to the first two kinds of business average scheduling delay which is higher. The PF algorithm takes the characteristics of the priority into account, the 
lower priority of the business with higher priority, but still cannot meet the QoS requirements of the service in the power grid. Compared with the traditional PF and RR algorithms, the delay of the first and second types of higher priority is obviously lower than that of the traditional PF and RR algorithm. This is because the algorithm has recalculated the priority before each scheduling sorting. So the first two types of business can be arranged to the front of the scheduling team and scheduled for priority, to ensure that the required service delay can be satisfied.

\section{Conclusion}

In this paper, a dynamic uplink resource scheduling algorithm based on SDON is proposed for power wireless private network, which is improved on the basis of the traditional $\mathrm{PF}$ algorithm. Considering the business characteristics of the power wireless private network, the QoS requirement of business is ensured by the SDON resource management platform allocation resource based on service priority. Simulation results show that the proposed algorithm is more suitable for the communication in power wireless private network. Under the constraints of uplink resource allocation in TD-LTE system, the algorithm proposed in this paper can guarantee the best uplink transmission rate of terminals, improve the system throughput, and meet the complex QoS requirements of business in power wireless private network.

\section{Conflicts of Interest}

The author declares that he has no conflicts of interest.

\section{Acknowledgments}

This work was supported by State Grid Science and Technology Project 52018E15000G.

\section{References}

[1] L. Xiaoli, "Research on the planning and evolution of communication network based on smart grid service," China Science and Technology Information, vol. Z1, pp. 85-87, 2015.

[2] I. Tomkos, F. Effenberger, and J.-K. K. Rhee, "Introduction to the special issue on optical networking for $5 \mathrm{G}$ mobile and wireless Communications," Journal of Optical Communications and Networking, vol. 8, no. 12, pp. FGM1-FGM4, 2016.

[3] A. Irfan, N. Taj, and S. A. Mahmud, "A novel secure SDN/LTE based architecture for smart grid security," in Proceedings of the 15th 2015 IEEE International Conference on Computer and Information Technology; Ubiquitous Computing and Communications; Dependable, Autonomic and Secure Computing; Pervasive Intelligence and Computing, pp. 762-769, Liverpool, UK, October 2015.

[4] S.-B. Lee, I. Pefkianakis, A. Meyerson, S. Xu, and S. Lu, "Proportional fair frequency-domain packet scheduling for $3 \mathrm{GPP}$ LTE uplink," in Proceedings of the 28th Conference on Computer Communications (IEEE INFOCOM '09), pp. 2611-2615, Rio de Janeiro, Brazil, April 2009.

[5] Z. Songlin, G. Yong, and Y. Yongjia, "ZTE's perspective on applying OFDM-PON in next converged optical and wireless networks," China Communications, vol. 12, no. 4, pp. 50-57, 2015.

[6] W. Ji and Z. Kang, "Design of WDM RoF PON based on OFDM and optical heterodyne," Journal of Optical Communications and Networking, vol. 5, no. 6, pp. 652-657, 2013.

[7] Y. Shao, K. Wang, S. Qinghua et al., "Study on on communication technique in the environment of smart grid," Guangdong Electric Power, vol. 11, pp. 52-55+73, 2011.

[8] X. Cao, N. Yoshikane, I. Popescu, T. Tsuritani, and I. Morita, "Software-defined optical networks and network abstraction with functional service design [invited]," Journal of Optical Communications and Networking, vol. 9, no. 4, pp. C65-C75, 2017.

[9] S. Jian-ping, "LIN Chang-cone.Study on communication technology of intelligent distribution network based on TD-LTE," Power System Communications, vol. 33, no. 7, pp. 80-83, 2012.

[10] Z. Ge, R. Gu, and Y. Ji, "An active queue management adaptation framework for software defined optical network," in Proceedings of the 2014 13th International Conference on Optical Communications and Networks, ICOCN 2014, Suzhou, China, November 2014.

[11] Q / GDW 513-2010, Power distribution automation system master function specification.

[12] X. Duan, A. M. Akhtar, and X. Wang, "Software-defined networking-based resource management: data offloading with load balancing in 5G HetNet," Eurasip Journal on Wireless Communications and Networking, vol. 2015, no. 1, article 181, 2015.

[13] Q/GDW_373-2009, Power users electricity information collection system functional specifications.

[14] C. E. Koksal, H. Kassab, and H. Balakrishnan, "An analysis of short-term fairness in wireless media access protocols (poster session)," ACM SIGMETRICS Performance Evaluation Review, vol. 28, no. 1, pp. 118-119, 2000.

[15] R. Guerzoni, R. Trivisonno, and D. Soldani, "SDN-based architecture and procedures for 5G networks," in Proceedings of the 2014 1st International Conference on 5G for Ubiquitous Connectivity, 5GU 2014, pp. 209-214, November 2014.

[16] J. Brown and J. Y. Khan, "Performance analysis of an LTE TDD based smart grid communications network for uplink biased traffic," in Proceedings of the 2012 IEEE Globecom Workshops, GC Wkshps 2012, pp. 1502-1507, Anaheim, Calif, USA, December 2012.

[17] C. Siqian, "Research on radio resource scheduling algorithm in LTE system," Harbin Institute of Technology, pp. 120-135, 2010.

[18] L. Chen, J. Lu, and C. India, "LTE based on QoS business proportional fair scheduling algorithm," Optical Communication Research, vol. 5, pp. 64-67, 2012. 

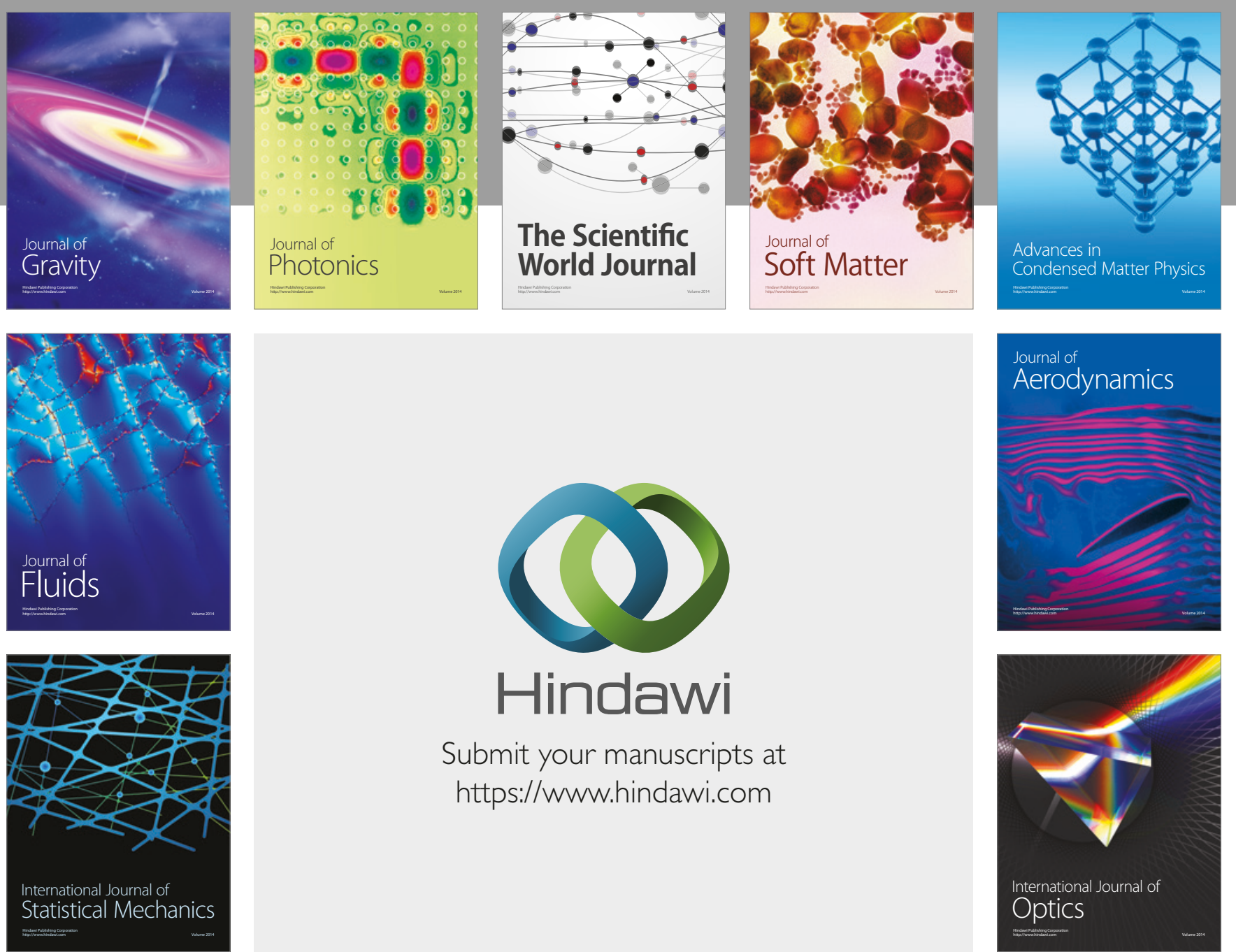

Submit your manuscripts at

https://www.hindawi.com
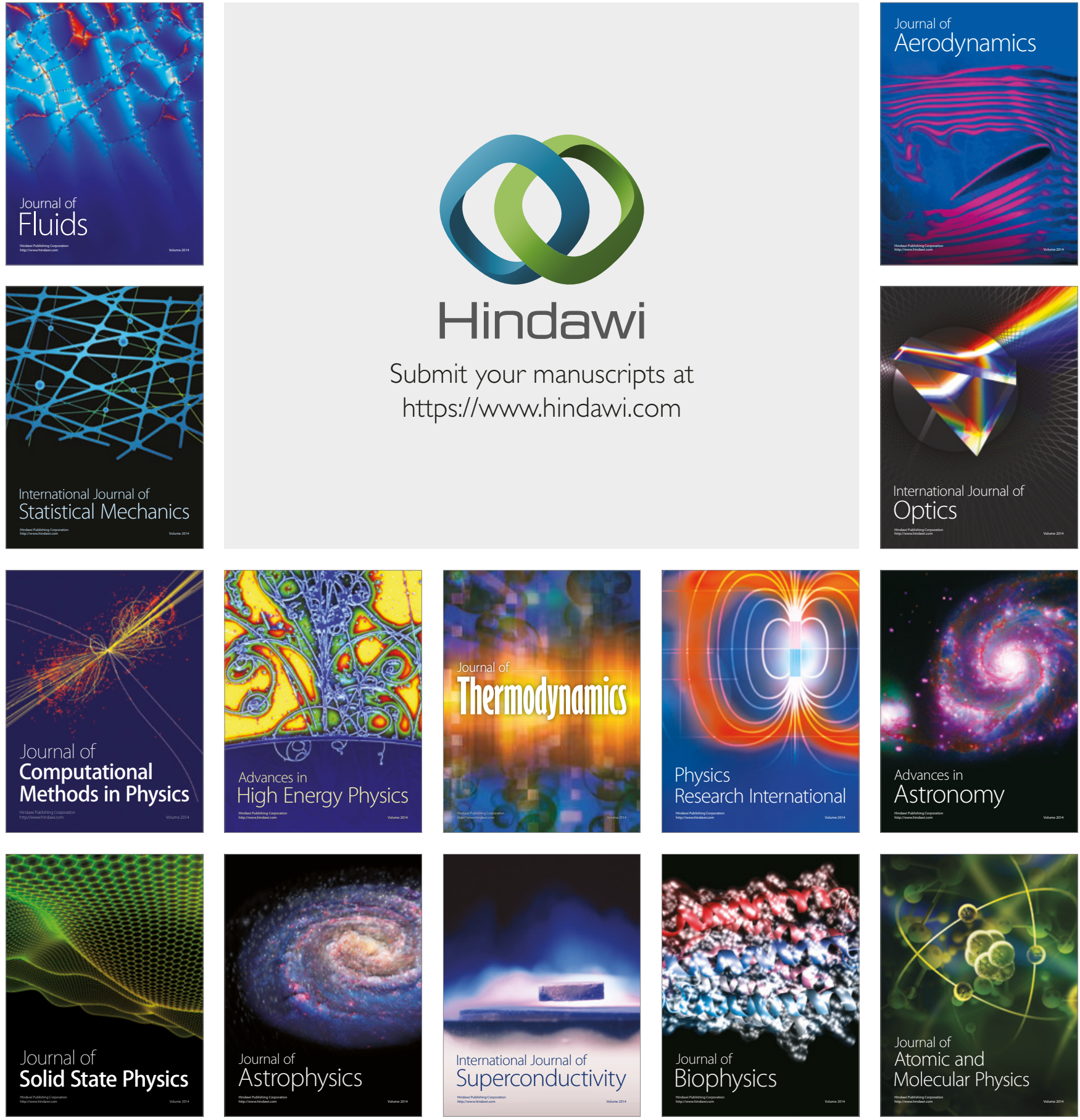